Bio - grafia. Escritos sobre la Biología y su Enseñanza. ISSN 2027

Edición Extraordinaria. p.p. 355 - 365

Memorias del VIII Encuentro Nacional de Experiencias en Enseñanza de la Biología y la Educación Ambiental. III Congreso Nacional de Investigación en Enseñanza de la Biología.

\title{
Mitos del agua y conservación: Planteamiento de un problema y revisión de antecedentes
}

Water myths and conservation: Approach a problem and antecedents revision

Gina Paola Ojeda Gonzalez ${ }^{1}$

El agua es un elemento vital para la supervivencia del hombre y demás seres vivos (CAR, 2001), todas las personas la utilizan en el día a día, está presente en los alimentos que consumen, en los materiales que manipulan, en las condiciones climáticas que afectan los lugares que frecuentan, en el cuerpo, en los ríos, riachuelos, humedales y lagunas que recorren la ciudad, en los ecosistemas que cubren gran parte del planeta, en las nubes, en el aire, en los recuerdos de vida y, en las historias de los pueblos y de su gente. La mística y la espiritualidad del agua tienen su motivación más profunda en la defensa de la vida, ya que no existe vida sin agua y todas las formas de vida dependen de ella. De esta forma, el agua adquiere un valor vital y sagrado: Bien común, patrimonio de la humanidad y derecho de todas y todos (Ferro, 2006). Colombia, con su ubicación geográfica, su variada topografía y sus condiciones climatológicas características del neotrópico, es uno de los países con mayor riqueza hídrica del planeta, como lo afirma García et al. (1998), superando una escorrentía de $2100 \mathrm{~km}^{3}$ anualmente (IDEAM, 2004, pp. 55). El agua que se distribuye diariamente en la ciudad de Bogotá, como lo afirma Ortiz y Reyes en el 2009, proviene en un $80 \%$ del páramo de Chingaza y un $20 \%$ del páramo de Sumapaz, ecosistemas naturales que no se encuentran protegidos totalmente dentro de los Parques Nacionales Naturales y, por lo tanto, están expuestos a una alta explotación económica trayendo como consecuencia un grave daño ambiental y ecológico. Daño que también impacta a los habitantes citadinos, para quienes el agua es un recurso de uso diario mayoritariamente para las labores domésticas.

${ }^{1}$ Estudiante noveno semestre Licenciatura en Biología, Universidad Pedagógica Nacional. E-mail: elamorylapureza@hotmail.com 
Bio - grafia. Escritos sobre la Biología y su Enseñanza. ISSN 2027

Edición Extraordinaria. p.p. 355 - 365

Memorias del VIII Encuentro Nacional de Experiencias en Enseñanza de la Biología y la Educación Ambiental. III Congreso Nacional de Investigación en Enseñanza de la Biología.

Recorrer día a día la ciudad de Bogotá, deja ver claramente que los cuerpos de agua que la atraviesan son contaminados $y$, un gran porcentaje de los afluentes del río Bogotá, presentan calidad de agua "Pobre" y "Marginal" según las categorías del WQI en un informe de la Secretaría Distrital de Ambiente (2013). Una de las causas del mal estado de las aguas son los diferentes tipos de residuos sólidos como envolturas de comidas, plásticos, papel, metal, restos biológicos y, residuos líquidos como los aceites y los lixiviados provenientes del relleno sanitario de Doña Juana que llegan directamente a las aguas del río Tunjuelo. A su vez, el río Bogotá se contamina por "miles de toneladas de desechos orgánicos que recibe de alcantarillados y fábricas que lo envenenan con toda clase de residuos como el arsénico, las grasas, plásticos, caucho, cobre y mercurio" (Prieto, 2004, pp. 256). Adicionalmente, los terrenos que corresponden a los humedales capitalinos han sido enterrados bajo grandes edificios rascacielos de uso habitacional, a pesar de que el Ministerio de Ambiente en su Política Nacional para Humedales Interiores de Colombia del año 2001, "Propende por la conservación y el uso racional de los humedales interiores con el fin de mantener y obtener beneficios ecológicos, económicos y socioculturales" IDEAM (2004, pp 242).

Este mal estado de los ecosistemas acuáticos bogotanos ha llevado a que los habitantes de la ciudad tengan poca importancia por el estado de sus cuerpos hídricos, generando una cultura con una grave falta de apropiación de sus recursos hídricos a pesar de las diversas campañas de conservación y uso sostenible que se realizan constantemente desde las distintas administraciones distritales "hace más de 20 años" (Alcaldía Mayor de Bogotá, 2011, pp. 10).

Dado que la mayoría de las comunidades indígenas que habitan en Colombia se encuentran en territorios con una alta biodiversidad y bajos niveles de contaminación, como es el caso de la Región Amazónica que hidrográficamente abarca las cuencas de los ríos Caquetá, Putumayo, Vaupés y Guainía con una escorrentía media de 4200mm (IDEAM, 2004) y que además, cuenta con la presencia de 44 pueblos indígenas según datos del Departamento Nacional de Planeación en 1997 (Arango, 1997), se hace importante indagar en torno a los mitos referentes al agua, como elementos de la cultura que se relacionan directamente con las distintas formas de actuar de las personas y que, con el paso del tiempo, han sido 
Bio - grafia. Escritos sobre la Biología y su Enseñanza. ISSN 2027

Edición Extraordinaria. p.p. 355 - 365

Memorias del VIII Encuentro Nacional de Experiencias en Enseñanza de la Biología y la Educación Ambiental. III Congreso Nacional de Investigación en Enseñanza de la Biología.

olvidados por las generaciones venideras, haciendo cada vez más creciente la pérdida de la endoculturación y el olvido de sus historias ancestrales, posiblemente, debido a que la cultura occidental ha permeado la indígena y ha promovido la creencia de la "invalidez" de lo mítico y lo espiritual.

Las comunidades indígenas tienen un gran conocimiento de su territorio; en cuanto a la vegetación, como lo asegura la Comisión Amazónica de Desarrollo y Medio Ambiente en 1994, ellos identifican las especies de árboles adultos, juveniles y semillas, así como las propiedades de las raíces, cortezas, maderas, hojas, savias, resinas, flores y frutos de diferentes especies. Adicionalmente, dichas comunidades tienen una gran cantidad de conocimientos en torno a la fauna tanto terrestre, como arbórea y acuática, dependiendo del asentamiento geográfico de la población. La ubicación geográfica de las comunidades incide directamente en sus interacciones con el agua, ya que éstas son diferentes en áreas de varzea, tierra firme, zona de inundación y piedemonte andino. Es así, como el olvido de los saberes ancestrales de las comunidades indígenas de Colombia, y del resto del mundo, traería como consecuencia una gran pérdida de conocimiento en torno a la biodiversidad y el manejo sostenible de los recursos naturales, lo que resalta la importancia de la recuperación y divulgación de estos saberes indígenas, y acelera la preocupación en los ámbitos académicos por investigar más a fondo su cosmovisión y sus costumbres.

En lo que respecta a la sociedad y al ámbito educativo, es necesario generar cambios en las actitudes, acciones y pensamientos de los habitantes citadinos y que, además aporten al proceso de construcción de una cultura ciudadana que sea defensora de los ecosistemas naturales y que propenda por el manejo adecuado del agua en diferentes contextos, aportando a la recuperación y mantenimiento de cada una de las fuentes de agua que recorren Bogotá. Es necesario que los humanos entiendan que no existe el mundo como un "objeto" puesto frente a ellos, sino que hacen parte de él, formando una unidad, es indispensable retomar y recuperar ese pensamiento ancestral que aporta un fuerte sustento para la modificación de ciertas prácticas desfavorables para la conservación del planeta Tierra. 
Bio - grafia. Escritos sobre la Biología y su Enseñanza. ISSN 2027

Edición Extraordinaria. p.p. 355 - 365

Memorias del VIII Encuentro Nacional de Experiencias en Enseñanza de la Biología y la Educación Ambiental. III Congreso Nacional de Investigación en Enseñanza de la Biología.

Los mitos son un elemento de gran influencia en la mentalidad de los hombres y generador de estrechas relaciones con su medio circundante. La recopilación de los mitos del agua en Colombia pretende rescatar las nociones ancestrales del agua que tienen las diferentes culturas indígenas colombianas, llamar la atención de las generaciones venideras y, aportar al cambio de paradigma de una visión inmediatista e individual, a una sistémica y ecológica.

Desde la formación como Licenciada en Biología, es de suma importancia el reconocimiento de las cosmovisiones de las múltiples comunidades indígenas que habitan el territorio colombiano, articulando dicho pensamiento con las relaciones hombre - naturaleza que se han visto olvidadas en la cultura citadina. Esto, con la finalidad de indagar, conocer y construir caminos que posibiliten la enseñanza del cuidado y conservación de los ecosistemas naturales en contextos urbanos como la ciudad de Bogotá. Además, es importante que los Licenciados en Biología asuman el rol del maestro como un contador de historias, que lleguen a la subjetividad del pensamiento de cada individuo al que son contadas y que trasciendan en ellos para convertirse en acciones diarias que promuevan el cuidado de la naturaleza y, en este caso, de los ecosistemas acuáticos.

Con el objetivo de indagar sobre las investigaciones realizadas hasta el momento en las temáticas concernientes a los mitos, el agua y la conservación, se recopilan los antecedentes encontrados a nivel local, nacional $e$ internacional. Desde el ámbito internacional se resaltan cuatro investigaciones, una de ellas realizada en el año 2006, relatada en un libro titulado "La Cultura del Agua: Lecciones de la América Indígena", escrito por Vargas, R. en Uruguay y patrocinado por la UNESCO, en el que el objetivo principal es hacer aportes de la cultura del agua y brindar elementos analíticos para estudiar de una manera diferente la cuestión del agua, como por ejemplo el agua aborigen, la matriz de necesidades y satisfactores, las aguas mínimas y la no agua, logrando una contribución a la construcción de un paradigma diferente en la gestión del agua al repensar una nueva relación agua-sociedad, donde se revalore la importancia cultural y sagrada del agua, su función como integradora de comunidades, su noción como bien social y colectivo, entre otras. Este libro es una amplia visión latinoamericana del agua, que deja de verla sólo como un recurso, para verla como ser vivo, como ser divino, como derecho universal y 
Bio - grafia. Escritos sobre la Biología y su Enseñanza. ISSN 2027

Edición Extraordinaria. p.p. 355 - 365

Memorias del VIII Encuentro Nacional de Experiencias en Enseñanza de la Biología y la Educación Ambiental. III Congreso Nacional de Investigación en Enseñanza de la Biología.

comunitario, posibilitando el cuestionamiento de las formas de pensamiento lineales y abriendo camino a nuevas concepciones.

Otro trabajo investigativo internacional que se destaca, es una iniciativa de resignificación del agua, llevada a cabo anualmente desde el año 2010 en diferentes ciudades del mundo, llamada "Canto al agua", es un canto real y consciente del mantra "AH", cantado con la energía de amor, agradecimiento y perdón hacia las aguas del planeta, que se realiza todos los 22 de Marzo (Día Mundial del Agua), en diferentes puntos geográficos a las $12 m$ - hora local. Es un canto que nace desde cada corazón, con la intención de sanar las aguas de cada territorio para que sumados puedan despertar nuestra consciencia y sanar las aguas del planeta entero. El evento "Canto al agua" vincula la dimensión ambiental del agua con la dimensión mística de los mantras, en pro de la recuperación de los cuerpos de agua presentes en las diferentes ciudades del mundo, utilizando la espiritualidad de cada persona para establecer conexiones con el agua y sentir esa unidad con la madre Tierra.

En el año 2012, Gómez, S., Aranguren, J. \& Luigi, M., realizaron una investigación titulada "Valores ambientales en el mito de Maria Lionza", desarrollado en la ciudad de Yaracuy, Venezuela. Éste trabajo tuvo como objetivo general "Identificar los valores ambientales presentes en distintas versiones del mito de María Lionza, y así obtener su base axiológica para entender las acciones de los creyentes del culto durante sus prácticas sociales y mítico-religiosas", encontrando como resultado que desde la educación ambiental se puede fortalecer la identidad, los valores, las creencias y la vinculación con el ambiente al utilizar el mito como herramienta educativa; además, los valores encontrados en el mito de María Lionza llevados a la praxis, permiten formar seres humanos íntegros, basados en una ética ambientalmente responsable donde se respete el bienestar propio, del prójimo y del entorno, es decir, estos valores pueden fomentar actitudes y comportamientos en búsqueda de la sustentabilidad. Esta investigación reconoce el mito como un potente influenciador de la cultura y de las acciones de los individuos creyentes en él, lo que viabiliza el mito como una valiosa herramienta educativa que puede ser ampliamente estudiada, difundida $e$ implementada. 
Bio - grafia. Escritos sobre la Biología y su Enseñanza. ISSN 2027

Edición Extraordinaria. p.p. 355 - 365

Memorias del VIII Encuentro Nacional de Experiencias en Enseñanza de la Biología y la Educación Ambiental. III Congreso Nacional de Investigación en Enseñanza de la Biología.

Finalmente, se retoma el análisis investigativo realizado en España por Emilio Chuvieco en el año 2012, titulado "Religious approaches to water management and environmental conservation" publicado en la revista Water Policy. El autor realizó un amplio abordaje de los textos "sagrados" de algunas de las religiones más seguidas por las personas en el mundo (Cristianismo, Judaísmo, Islamismo, Hinduismo y Budismo), analizando los principios éticos y morales allí explicitados con relación con el ambiente y en especial las relaciones hombre-naturaleza-Dios. El autor logró identificar en la mayoría de las religiones una tendencia "teocéntrica", que guía las acciones de sus seguidores hacia una alabanza y una honra a su Dios, dejando de lado el ambiente y la naturaleza que "Dios ha creado para ellos"; los textos religiosos hablan del hombre como "amo" y "guardián" de la naturaleza, no como "explotador" y "destructor", acciones que no son llevadas a cabo por muchos de los seguidores. El autor asegura que las religiones son un importante elemento para soportar actitudes hacia el ambiente, en cuanto cada religión establece principios para actuar desde lo ético y lo moral, lo que hace que las creencias religiosas sean una importante temática para investigar y generar acciones en pro de la conservación del ambiente.

Cambiando de ámbito, a nivel nacional se han encontrado varias investigaciones entre las que se encuentra un estudio investigativo realizado por Hugo Portela en el año 2000, patrocinado por la Universidad del Cauca y, titulado "El pensamiento de las aguas de las montañas: Coconucos, Guambianos, Paeces, Yanaconas". El tema central del libro es la interpretación de los territorios de gran riqueza acuática y la importancia del agua en el pensamiento y la comprensión del mundo de cuatro grupos indígenas del suroccidente de Colombia. En el libro se analizan las clasificaciones del agua y las relaciones humanos - agua y, humanos - medio acuático que posibilitan la armonía, el equilibrio y el bienestar individual y colectivo. Este estudio aporta una visión desde la antropología de las relaciones que se establecen entre las creencias y normas establecidas desde los pueblos indígenas con el uso y cuidado del agua que atraviesa los territorios de cuatro de las comunidades indígenas del país, lo que apoya la premisa de la influencia que puede llegar a tener el conocimiento mítico y espiritual de las culturas indígenas ancestrales sobre el pensamiento y comportamiento de los humanos. 
Bio - grafia. Escritos sobre la Biología y su Enseñanza. ISSN 2027

Edición Extraordinaria. p.p. 355 - 365

Memorias del VIII Encuentro Nacional de Experiencias en Enseñanza de la Biología y la Educación Ambiental. III Congreso Nacional de Investigación en Enseñanza de la Biología.

Igualmente, a nivel nacional se resalta un trabajo investigativo realizado en el año 2005 por la Fundación Zoológica de Cali y el Centro de Investigación para la Conservación de Especies Amenazadas (CREA), a través del proyecto "Lobo de Río", titulada "La gente de agua: Una travesía por el río Apaporis", en el que pretenden resaltar el valor de los ríos y humedales de Colombia, dando a conocer la vital importancia que representan para algunas culturas de la Amazonía y Orinoquía de nuestro país, los ecosistemas acuáticos. El producto del trabajo es un homenaje a todas las culturas que desde su sabiduría reconocen el gran valor que tienen los ríos y los seres vivos que los habitan porque gracias a ellos es que se logrará su conservación. Esta investigación aporta la importancia de indagar en torno a las relaciones existentes entre las comunidades humanas y los ecosistemas acuáticos y, a partir de allí proponer caminos para el abordaje de temáticas referentes al cuidado y conservación de la madre Tierra.

También se retoma la investigación realizada en el año 2006 por Ferro, A., titulado "La dimensión sagrada, simbólica y mística del agua", y publicado en la Revista Semillas No. 28. Su objetivo principal fue "Argumentar la importancia del agua en las comunidades indígenas, campesinas y citadinas, definiéndola como la vida misma y como patrimonio de la humanidad". El estudio fue realizado basándose en la revisión documental y en las vivencias propias, resaltando el valor biológico, social, simbólico y espiritual, paisajístico y turístico, artístico y poético y, ecológico del agua; resaltando que el agua posee un fuerte simbolismo y una dimensión sagrada en las culturas desde varias dimensiones y significados de la vida humana que deben ser tratados en los proyectos que incluyen a la comunidad, pero no como mano de obra sino eje fundamental. Este documento es importante, dado que en él se refleja todo el simbolismo que tiene el agua para las diversas comunidades, brindando una amplia gama de razones por las que el agua debe ser cuidada y conservada por todas las personas desde el conocimiento indígena y ancestral.

En lo concerniente a las investigaciones a nivel local, se retoma el trabajo de investigación realizado por Navarro, O. durante el año 2004 en la ciudad de Bogotá, titulado "Representación social del agua y sus usos", cuyos objetivos fueron "Identificar la representación social que tiene un grupo social colombiano respecto al agua y, Reconocer los elementos que constituyen la representación social del agua en la población 
Bio - grafia. Escritos sobre la Biología y su Enseñanza. ISSN 2027

Edición Extraordinaria. p.p. 355 - 365

Memorias del VIII Encuentro Nacional de Experiencias en Enseñanza de la Biología y la Educación Ambiental. III Congreso Nacional de Investigación en Enseñanza de la Biología.

entrevistada, así como la estructura que la organiza, para confrontarlas con las declaraciones hechas acerca de los usos e intereses sobre la misma". La recolección de la información se realizó mediante entrevistas estructuradas a jóvenes universitarios, encontrando que los problemas ambientales ocupan un lugar importante dentro de las preocupaciones de la población entrevistada, y que, entre lo que los sujetos declaran o "dicen hacer" y lo que "hacen" realmente existe una brecha, un vacío entre la orientación de intereses (actitud) y las acciones (comportamiento). Este estudio aporta una caracterización social de un grupo focal de universitarios en Bogotá en temáticas referentes al agua, proporcionando un panorama general de las creencias y actitudes que presenta una parte de la población citadina.

Otro trabajo investigativo que se resalta con la comunidad citadina se titula "Memorias del Agua en Bogotá: Antología", publicado en el año 2011 y patrocinado por la BLAA, la Pontificia Universidad Javeriana y la Alcaldía Mayor de Bogotá - Bogotá Positiva. Esta investigación tuvo como objetivo general recoger historias que reflejaran las relaciones cotidianas de los capitalinos con el agua como recurso indispensable; historias sobre lugares, personajes, oficios, prácticas, ritos, leyendas y usos del agua en el pasado y en el presente, mediante talleres de creación literaria. Dicho trabajo investigativo permite conocer y caracterizar parte de la comunidad bogotana. Adicionalmente, aporta a la construcción de memoria colectiva partiendo de relatos de vivencias individuales con el propósito de consolidar una cultura de la memoria y de las historias que surgen en torno al agua en la cotidianidad de Bogotá.

Otro aporte a nivel local lo realiza Ruíz, A. \& Flórez, M. en el año 2015, para optar por el título de Licenciadas en Biología de la Universidad Pedagógica Nacional, titulado "El cuento como material didáctico en la enseñanza de los valores de la conservación del agua en primera infancia", cuyo objetivo general fue Construir un cuento para la primera infancia como propuesta para la apropiación de los valores enfocados a la conservación del agua. Como resultados de este trabajo se encontró que es importante fomentar el cuidado del agua por medio de valores de conservación a niños de primera infancia ya que estos son sujetos de cambio dentro de una sociedad. Este trabajo aporta la importancia de la 
Bio - grafia. Escritos sobre la Biología y su Enseñanza. ISSN 2027

Edición Extraordinaria. p.p. 355 - 365

Memorias del VIII Encuentro Nacional de Experiencias en Enseñanza de la Biología y la Educación Ambiental. III Congreso Nacional de Investigación en Enseñanza de la Biología.

narrativa y de su uso para la enseñanza de los valores de conservación en la primera infancia.

Cada uno de los antecedentes mencionados anteriormente consolidan un camino en torno a los mitos del agua, las concepciones de la gente sobre el agua, las cosmovisiones indígenas del agua $y$, las posibles influencias de la cultura en el manejo del recurso hídrico, camino que ha empezado a ser recorrido por diversos investigadores y al que se pretende hacer una importante contribución. La continua exploración en torno a las cosmovisiones indígenas de la naturaleza y su posible uso en la enseñanza de la conservación de ecosistemas en contextos citadinos, debe ser una importante corriente de investigación en el campo de la enseñanza de la Biología, con el objetivo de contribuir a la construcción de paradigmas de vida tendientes al ecocentrismo $y$, aportar a la consolidación de una sociedad más consciente y menos indiferente a las problemáticas ambientales que atraviesa su ciudad, resaltando la importancia que tienen las acciones que cada individuo realiza en su día a día.

\section{Bibliografía}

- Alcaldía Mayor de Bogotá. (2011). Memorias del Agua en Bogotá: Antología. Bogotá, Colombia: Imprenta Distrital.

- Arango, R. \& Sánchez, E. (1997). Los Pueblos Indígenas de Colombia - Desarrollo y Territorio. Colombia: TM Editores \& Departamento Nacional de Planeación.

- Canto al agua. (2010). ¿Qué es Canto al agua?. Recuperado de http://www.cantoalagua.com/\#!que-es-cantoalagua/cleew el 19 de Marzo de 2015.

- Chuvieco, E. (2012). Religious approaches to water management and environmental conservation. Water policy, 14, pp. 9-20.

- Comisión Amazónica de Desarrollo y Medio Ambiente. (1994). Amazonía sin mitos. Colombia: Editorial Oveja Negra.

- Corporación Autónoma Regional (CAR). (2001). Atlas Ambiental de Cundinamarca. Primera edición. Colombia: CAR.

- Ferro, A. (2006). La dimensión sagrada, simbólica y mística del agua. Revista Semillas, (28), pp. 35-38. 
Bio - grafia. Escritos sobre la Biología y su Enseñanza. ISSN 2027

Edición Extraordinaria. p.p. 355 - 365

Memorias del VIII Encuentro Nacional de Experiencias en Enseñanza de la Biología y la Educación Ambiental. III Congreso Nacional de Investigación en Enseñanza de la Biología.

- Fundación Zoológica de Cali \& Centro de Investigación para la Conservación de Especies Amenazadas (CREA). (2005). La Gente de Agua: Una travesía por el río Apaporis. Cali, Colombia: Fundación Zoológica de Cali.

- García, M., et al. (1998). El Agua. En: P. Leyva (Ed.), El Medio Ambiente en Colombia (Capítulo 4). Bogotá: Instituto de Hidrología, Meteorología y Estudios Ambientales.

- Gómez, S., Aranguren, J. \& Luigi, M. (2012). Valores ambientales en el mito de Maria Lionza. Revista de Investigación, 36, (77), pp. 165 - 184. Recuperado el 24 de Noviembre de 2014 de la base de datos Scielo.

- IDEAM. (2004). Informe Anual sobre el Estado del Medio Ambiente y los Recursos Naturales Renovables en Colombia. Colombia: IDEAM.

- Navarro, O. (2004). Representación social del agua y sus usos. Psicología desde el caribe, (4), $222-236$.

- Ortiz, L. \& Reyes, M. (2009). Páramos en Colombia: Un ecosistema vulnerable. Revista Observatorio Grupo de Estudios en Economía Política y Medio Ambiente. Recuperado de la página http://www.usa.edu.co/observatorio_economico/Observatorio\%20Ambiental/param os-colombia.pdf el 3 de Marzo de 2015.

- Portela, H. (2000). El pensamiento de las aguas de las montañas: Coconucos, Guambianos, Paeces, Yanaconas. Cali, Colombia: Editorial Universidad del Cauca.

- Prieto, C. (2004). El agua: Sus formas, Efectos, Abastecimientos, Usos, Daños, Control y Conservación. Bogotá, Colombia: Ecoe Ediciones.

- Ruíz, A. \& Flórez, M. (2015). El cuento como material didáctico en la enseñanza de los valores de la conservación del agua en primera infancia. Tesis para optar por el título de Licenciadas en Biología no publicada. Universidad Pedagógica Nacional, Bogotá, Colombia.

- Secretaría Distrital de Ambiente. (2013). Informe Sectorial Secretaría Distrital de Ambiente Vigencia 2012 Indicadores de Gestión de la Administración Distrital Acuerdo 067 de 2002 Indicadores de Impacto Acuerdo 489 de 2012 Plan Distrital de Desarrollo 2012 - 2016 "Bogotá Humana". Recuperado el 03 de Marzo de 2015 de 
Bio - grafia. Escritos sobre la Biología y su Enseñanza. ISSN 2027

Edición Extraordinaria. p.p. 355 - 365

Memorias del VIII Encuentro Nacional de Experiencias en Enseñanza de la Biología y la Educación Ambiental. III Congreso Nacional de Investigación en Enseñanza de la Biología.

http://ambientebogota.gov.co/c/document_library/get_file?uuid=fac23731-440c4f40-8f5b-1d59d0afdb5b\&groupId=55886.

- Vargas, R. (2006). La Cultura del Agua: Lecciones de la América Indígena. Uruguay: Programa Hidrológico Nacional UNESCO. 